Research Article

\title{
Emergency Resource Location and Allocation in Traffic Contingency Plan for Sports Mega-Event
}

\author{
Ling Shen $\mathbb{D}^{1},,^{1,2,3}$ Jian Lu $\mathbb{D}^{1},{ }^{1,2,3}$ Ling Deng, ${ }^{1,2,3}$ and Manman $\mathrm{Li}^{4}$ \\ ${ }^{1}$ Jiangsu Key Laboratory of Urban ITS, Southeast University, Nanjing 21189, China \\ ${ }^{2}$ Jiangsu Province Collaborative Innovation Center of Modern Urban Traffic Technologies, Southeast University, \\ Nanjing 211189, China \\ ${ }^{3}$ School of Transportation, Southeast University, Nanjing 211189, China \\ ${ }^{4}$ School of Automoblie, Chang'an University, Xi'an 710054, China \\ Correspondence should be addressed to Jian Lu; lujian_1972@seu.edu.cn
}

Received 22 November 2020; Revised 16 March 2021; Accepted 15 April 2021; Published 24 April 2021

Academic Editor: Hailing Kong

Copyright (c) 2021 Ling Shen et al. This is an open access article distributed under the Creative Commons Attribution License, which permits unrestricted use, distribution, and reproduction in any medium, provided the original work is properly cited.

In view of the transactional and textual features on issue handling in mega-event traffic contingency plan, this paper gives a quantitative method for emergency resources location and allocation. Given that the requirement on safeguards in the sports mega-events is temporary and stringent, we first divide the facilities into temporary emergency facilities and fixed emergency facilities and the resources into material resources and human resources. Considering the uncertainty of emergency incidents, we then construct a mixed integer linear programming model. To solve this model, the bisection method is used to import the material quantity placed in each emergency facility, and the shortest path algorithm is used to import the rescue time matrix. Considering the slowness of convergence rate when the road network is large, a modified matrix real-coded genetic algorithm is designed with the crossover operator based on a greedy algorithm. The application of the model and algorithms is validated by the case based on 2022 Beijing Winter Olympics. Sensitivity analysis of some important parameters is also conducted to provide insights for traffic emergency resources management in sports mega-event.

\section{Introduction}

Mega-events about sports have extremely high requirements for the steady traffic operation that heavily relies on reasonable traffic emergency resource plan. Nowadays, traffic emergency resource plan is mainly designed by transactional and textual processing methods, easily bringing error. In addition, different from conventional emergency plans, the traffic emergency resource plan in mega-events about sports involves longer lines, wider cover, and various changes.

The conventional emergency resource plan consists of emergency facility locations and resource allocations with the objective of maximizing or optimizing the effective supply of emergency resources. With regard to emergency facility locations, a variety of models have been designed based on different emergency targets, service targets, and the nature of emergency events. But they can be divided into three categories: (1) $P$-center $[1,2]$, (2) coverage model [3, 4], and (3) $P$-median [5]. Table 1 analyzes the advantages, disadvantages, and applicable scenarios of these models and describes the advantages of the model in mega-events. On the basis of the classical algorithm, Jian et al. established emergency resource location model based on bandwidth allocation and location algorithm, respectively [6, 7]. Also, Yahyaei and Bozorgi-Amiri established a mixed integer linear programming model for material facility location problem [8]. Jun et al. considered the mutual influence of distribution location and resource delivery route to construct a facility location model and designed a discrete particle swarm optimization algorithm for it [9]. He and Liao simultaneously considered time and resource constraints to construct the facility location model [10]. Aiming at the problem of multiple disaster points in large-scale activities, Barbarosoglu and Arda established a game model from the 
perspectives of resource scheduling speed, cost function, resource demand and supply, and total cost function [11]. Das and Hanaoka established an AGENT-based multidisaster emergency resource allocation model considering time and distance [12]. Zhaoping and Jianping constructed a multiobjective emergency rescue resource allocation optimization model based on BURA by analyzing the process and influencing factors of emergency decision-making resource allocation, which reduces the complexity of emergency rescue [13]. Resources usually are allocated after facility location is chosen in literature, for example, Mete and Zabinsky [14]. However, the solution in this way is often suboptimal. To obtain optimal solution, Zhang et al. propose a dynamic multiobjective location-routing model to balance the responsiveness and the total response cost in emergency operations effectively [15].

From the literature review above, it is found that the existing emergency resource arrangement model does not consider characteristics of mega-event about sports and thus needs further to improve. The above literature only considers how to allocate materials economically and does not take into account the differences in the priority of large-scale activities. After the outbreak of special events, the material demand is largely related to the importance of relevant personnel. Measuring the emergency situation of large-scale activities and allocating materials are the focus of this paper. In mega-event about sports, the increase in traffic flow is sudden, so the temporary facilities are needed, besides fixed facility location. Second, both human resources and material resources are needed in dealing with traffic incident in megaevent about sports. Most previous studies just deal with a kind of resource. In addition, mega-events about sports have strict rescue time constricts and less focus on the money cost, which means that the air rescue or water channel rescue should be also included. Considering the three characteristics above, this paper established a new model and designed a new algorithm to comprehensively locate traffic emergency facilities and allocate traffic emergency resources in megaevent, with the objective of the efficient supply of emergency resources. Further, their application is validated by the 2022 Winter Olympics Case in Beijing.

\section{Model Development}

2.1. Problem Description and Assumption. This study focuses on the emergency resource location of the traffic operation during sport events, which responds to the coexistence of temporary traffic and conventional traffic security needs. Emergency resources are mainly various types of emergency rescue vehicles and equipment, including wreckers, pallet trucks, cranes, and fire trucks. Specifically, emergencies may break out in multiple locations with multiple requirements for emergency materials. Then the construction of appropriate emergency service facilities and the allocation of various emergency resources are needed. In order to put forward a targeted analysis of the problem, the establishment of the emergency resource arrangement model made the following established assumptions that are realistic:
(1) The construction cost of temporary emergency facilities is known.

(2) Any emergency demand point can accept rescue services provided by multiple emergency facilities.

(3) The construction costs of fixed emergency facilities are not considered, and the initial allocation of materials is assumed to be sufficient.

(4) When the ground rescue does not meet the time conditions, air rescue is dispatched, and it is assumed that the air rescue can deliver emergency supplies smoothly and on time without interference.

2.2. Variable and Parameter Definition. In order to describe the problem and develop the model better, Table 2 records the naming rules of sets, parameters, and decision variables used in model construction.

Among them, the value of $C_{j}$ should be determined according to the size of the actual facility and geographical location; $\operatorname{cap}_{j}^{k}$ is based on the specific scale of temporary facilities; the probability $\mu^{k}$ can be calculated according to the formula proposed by Church and Velle [16].

2.2.1. Material Weight. The demand for various emergency materials at facilities is closely related to the weight $W_{i}$ of the emergency demand points on each road section. On the one hand, the weight of each road section is related to the crash rate within a certain period. On the other hand, it is necessary to consider the priority of traffic operation guarantee during the sport mega-event. Even for a certain section with a low historical crash rate, if it is selected as a transportation channel for VIP personnel or materials, the level of emergency material support needs should be increased accordingly. Therefore, the method for determining the weight of material demand is as follows:

$$
W_{i}=A_{i} \cdot T_{i}
$$

where $A_{i}$ is the weight of road crash rate and $T_{i}$ is the weight of traffic service guarantee level. The calculation of $A_{i}$ is

$$
A_{i}=\frac{\omega_{i}}{\sum_{i \in I} \omega_{i}} \text {. }
$$

The crash rate in a certain period $\omega_{i}$ can be calculated by the following formula:

$$
\begin{aligned}
\omega_{i} & =\frac{P_{i}}{Q_{i}}, \\
P_{i} & =\sum_{s \in S} \theta_{s} p_{i s} .
\end{aligned}
$$

Among them, $P_{i}$ is the standard number of traffic accidents at emergency demand point $i, Q_{i}$ is the standard traffic volume of the road section $i, p_{i s}$ is the number of s-level traffic accidents on the road, and $\theta_{s}$ is the weighting coefficient of s-level accidents. The weighting coefficient can be obtained from the reference value in the following table [17]: reference value of weighting coefficient of accident level is shown in Table 3. 
TABLE 1: Comparison of emergency resource layout models.

\begin{tabular}{|c|c|c|c|}
\hline Model & Objective & Advantages and disadvantages & Applicable occasions \\
\hline$P$-median model & $\begin{array}{l}\text { Minimize weighted } \\
\text { distance }\end{array}$ & $\begin{array}{l}\text { Focus on efficiency, but sometimes } \\
\text { cannot meet emergency needs }\end{array}$ & $\begin{array}{c}\text { Nonemergency, places with relatively } \\
\text { fixed needs (parks, schools, and gas } \\
\text { stations) }\end{array}$ \\
\hline$P$-center model & $\begin{array}{l}\text { Minimize maximum } \\
\text { distance }\end{array}$ & $\begin{array}{c}\text { Emphasize on fairness, the number of } \\
\text { facilities to be built is determined, and } \\
\text { the coverage is not high }\end{array}$ & $\begin{array}{l}\text { High urgency and fairness-oriented } \\
\text { facilities (fire stations and police stations) }\end{array}$ \\
\hline Maximum coverage model & $\begin{array}{l}\text { Minimize the number of } \\
\text { facilities }\end{array}$ & $\begin{array}{c}\text { The number of facilities can be given, } \\
\text { and the degree of coverage is } \\
\text { maximized }\end{array}$ & $\begin{array}{l}\text { Nonurgent, high construction cost } \\
\text { occasions }\end{array}$ \\
\hline Set coverage model & $\begin{array}{r}\text { Maximiz } \\
\text { requir }\end{array}$ & $\begin{array}{c}\text { Cover all demand points, but lack } \\
\text { material allocation }\end{array}$ & $\begin{array}{l}\text { Timeliness requirements are high, and } \\
\text { emergency needs are relatively certain }\end{array}$ \\
\hline $\begin{array}{l}\text { Flexible location of } \\
\text { emergency resources for } \\
\text { sport mega-event model }\end{array}$ & $\begin{array}{l}\text { Minimize costs while } \\
\text { ensuring rescue time and } \\
\text { materials }\end{array}$ & $\begin{array}{l}\text { High emergency reliability, flexible } \\
\text { location, and hierarchical, but the cost } \\
\text { is difficult to control }\end{array}$ & $\begin{array}{l}\text { Volatile emergency demand, high } \\
\text { emergency reliability requirements, and } \\
\text { no consideration in cost occasion }\end{array}$ \\
\hline
\end{tabular}

TABLE 2: Model parameter naming table.

\begin{tabular}{|c|c|c|}
\hline Category & Symbol & Definition \\
\hline \multirow{5}{*}{ Set } & $I$ & Set of emergency demand points \\
\hline & $J$ & Set of candidates for temporary emergency facilities \\
\hline & $M$ & Set of supplies \\
\hline & $P$ & Set of fixed emergency facilities \\
\hline & $N_{i}$ & Set of facilities that can effectively cover emergency points \\
\hline \multirow{2}{*}{ Decision variables } & $X_{j}$ & (0-1 variable) whether to set up facilities at point $j$ \\
\hline & $y_{j}^{k}$ & Amount of materials $k$ placed in facility point $j$ \\
\hline \multirow{6}{*}{ Parameters } & $C_{j}$ & Construction cost of temporary facilities \\
\hline & $v^{k}$ & Volume of material $k$ \\
\hline & $q^{k}$ & Allocation cost of material $k$ \\
\hline & $t_{i j}$ & Rescue time from facility point $j$ to demand point $i$ \\
\hline & $S_{i}^{k}$ & The total amount of material $k$ covering demand point $i$ \\
\hline & $\operatorname{cap}_{j}^{k}$ & $\begin{array}{c}\text { Capacity of material } k \text { at facility point } j \\
\text { The probability of material } k\end{array}$ \\
\hline
\end{tabular}

TABLE 3: Reference value of weighting coefficient of accident levels.

\begin{tabular}{lccccc}
\hline & & \multicolumn{3}{c}{ Accident level s } \\
& Level 1 (serious) & Level 2 (major) & Level 3 (ordinary) & Level 4 (minor) \\
\hline Reference value $\theta_{s}$ & 11 & 5 & 1 & 0.33 \\
\hline
\end{tabular}

The traffic service guarantee level $T_{i}$ in this model is divided into three categories: $T_{1}, T_{2}$, and $T_{3}$. Among them, the main guarantee objects of $T_{1}$ level traffic are the President of the International Olympic Committee, the members of the International Olympic Committee, athletes, and team officials; $T_{2}$ level traffic is mainly guaranteed for staff, volunteers, contractors, and registered media participating in the event; $T_{3}$ level traffic is mainly guaranteed for members of the Olympic family and ticket holders audience. The weight reference values of various protection levels are shown in Table 4.

2.2.2. Reliability Constraints of Time. When deploying emergency resources for traffic operation during sport mega-event, the time reliability of emergency services is required. This model gives a time threshold $T$ and set $N$, a collection of emergency facilities that can provide services in time within the emergency rescue request time threshold $T \quad\left(N_{i}=\left\{\forall j \mid t_{i j}<T\right\}\right)$. Emergency demand points that exceed the rescue time requirements are served by fixed emergency facilities $P$ through air rescue. To ensure that all emergency services can reach the emergency demand point in time, the specific value of $T$ can be determined according to the scale and importance of the sport mega-event and the emergency guarantee requirements of the transportation route.

2.2.3. Reliability Constraints of Materials. In addition to the rigid requirements for emergency time, sufficient emergency supplies are also a necessary condition to ensure the operation of sport mega-event. Due to the uncertainty of emergencies during the sport mega-event, material guarantees must meet a certain level of service, $\alpha$. If the service 
TABLE 4: Reference value of traffic operation guarantee weight.

\begin{tabular}{|c|c|c|c|}
\hline & & $\begin{array}{l}\text { ration } \\
\text { riori }\end{array}$ & \\
\hline & $T_{1}$ & $T_{2}$ & $T_{3}$ \\
\hline Reference value $T_{i}$ & 1 & 0.8 & 0.6 \\
\hline
\end{tabular}

level $\alpha$ is set to 1 , it means that the necessary materials and equipment should be provided for emergency points with a $100 \%$ probability.

A certain material guarantee service level of emergency point $i$ should be positively related to material weight, namely, $\alpha \cdot W_{i}$.

For emergency point $i$, the total number of types of equipment on all emergency facilities covered by it is recorded as $S_{i}^{k}$, and then the probability of the guarantee that emergency point $i$ is provided with at least $\operatorname{dem}_{i}^{k}$ equipment $k$ is $\alpha \cdot W_{i}$. In other words, when a service request is made to an adjacent emergency reserve point, the probability of the number of devices $\operatorname{dem}_{i}^{k}$ in the idle state among all the above-mentioned devices should not be less than $\alpha \cdot W_{i}$.

Assuming that the probability of materials $k$ being occupied is $\mu_{k}$, the probability for idle state is $\left(1-\mu_{k}\right)$. For any emergency point $i$, the probability that the number of emergency materials not less than $\operatorname{dem}_{i}^{k}$ can be obtained in time is

$$
\begin{aligned}
f\left(\operatorname{dem}_{i}^{k}\right)= & C_{S_{i}^{k}}^{\operatorname{dem}_{i}^{k}}\left(1-\mu_{k}\right)^{\operatorname{dem}_{i}^{k}} \mu_{k}^{S_{i}^{k}-\operatorname{dem}_{i}^{k}} \\
& +C_{S_{i}^{k}}^{\operatorname{dem}_{i}^{k}+1}\left(1-\mu_{k}\right)^{\operatorname{dem}_{i}^{k}+1} \mu_{k}^{S_{i}^{k}-\operatorname{dem}_{i}^{k}-1} \\
& +\cdots+C_{S_{i}^{k}}^{S_{i}^{k}}\left(1-\mu_{k}\right)^{S_{i}^{k}} .
\end{aligned}
$$

With the expansion of supply, the probability of meeting demand will rise. Therefore, the above formula can be seen as a monotonically increasing function of the total number of types of covered equipment $S_{i}^{k}$. Therefore, when the value of $\alpha \cdot W_{i}$ is determined, $S_{i}^{k}$ that guarantees the reliability of the material will be the determined value.

\subsection{Temporary and Fixed Emergency Facilities}

2.3.1. Temporary Emergency Facilities Location Model. Temporary and fixed emergency facilities locations focus on different functions. The former are facilities added in the short term to meet the needs of traffic operation guarantee for sport mega-event. Under the premise of ensuring time constraints and material constraints, the optimization goal of the temporary emergency facilities location model is mainly for cost. The total cost of the emergency location system includes two aspects, the construction cost of temporary facilities and the allocation cost of materials. Therefore, the location optimization model of the temporary facility is

$$
\min C=\sum_{j=1} C_{j} X_{j}+\sum_{k \in K} \sum_{j \in J} y_{j}^{k} q^{k}
$$

Under the premise of ensuring emergency time and material reliability, the objective function is set to minimize the cost of the emergency system, where $\sum_{j=1} C_{j} X_{j}$ represents the total cost of building temporary facilities, and $\sum_{k \in K} \sum_{j \in J} y_{j}^{k} q^{k}$ represents the allocation cost of various materials in the facility.

Constraints are as mentioned above, which mainly restrict the time reliability of emergency response, material reliability, and allocation capacity of temporary facilities. The details are as follows:

s.t.

$$
\begin{aligned}
& \sum_{j \in N_{i}} y_{j}^{k} X_{j} \geq S_{i}^{k}, \quad \forall i \in I, k \in K, \\
& v^{k} y_{j}^{k} \leq \operatorname{cap}_{j}^{k} \cdot X_{j}, \quad \forall j \in J, \\
& 0 \leq y_{j}^{k} \leq \frac{\operatorname{cap}_{j}}{v_{k}}, \quad \forall j \in J, \forall k \in K, \\
& X_{j} \in\{0,1\}, \quad \forall j \in J, \\
& y_{j}^{k} \in N_{+}, \quad \forall j \in J, \forall k \in K .
\end{aligned}
$$

Constraints (7) aim at the reliability of emergency supplies. Among them, $j$ selects temporary emergency service facilities with emergency service time less than the maximum time threshold $t$. If the emergency rescue time threshold is exceeded, fixed facilities will be arranged to carry out rescue by air rescue. Constraints (8) means that only the points where emergency facilities are established can be rescued, and the volume of materials that can be provided should not exceed the allowable capacity of the facility; constraints (10) and (11) are the nonnegative limit and integer value limit of decision variables.

2.3.2. Fixed Emergency Facilities Location Model. In the context of mega-events, the function of fixed emergency facilities is to undertake general ground rescue and special air rescue missions. What is more, it should meet the need for material transfer between various facilities after the emergency point changes. The fixed emergency facilities adopt the form of screening the existing facilities; that is, the existing fixed emergency facilities in the research area are assembled and selected as alternative points, and their functions are transformed into fixed emergency facilities during sport mega-event. Facilities need to be screened in two aspects. One is to screen the scale of fixed emergency facilities. The small or nonair rescue facilities should be excluded. The other is to ensure that the total weighted distance between the fixed emergency facilities and the deployed temporary emergency facilities is less than the specified maximum distance. This is to guarantee the efficient transfer of materials and air rescue in the later period. At the same time, the minimum distance between fixed and temporary facilities should be restricted to prevent the close distance between different levels. The specific model is as follows:

$$
P=\left\{\forall p \mid \sum_{p \varepsilon P} \sum_{j \varepsilon J} d_{p j}<D_{\max }\right\},
$$


s.t.

$$
d_{p j}>D_{\text {min }}
$$

where $d_{p j}$ represents the distance from the existing facility $p$ to each temporary emergency facility. The conventional distance calculation method is the Euclidean distance; that is, the linear distance is obtained by the coordinates of two points. In this study, the specific transportation network distance was used instead of the Euclidean straight-line distance. This makes the simulation scenario of the model closer to reality, making the optimization result more accurate. The upper and lower limits of the distance threshold $D$ can be set according to the actual situation.

2.4. Comparison of Model Characteristics. Various emergency resource location models have different focuses. The model objectives, layout characteristics, and scope of application between the flexible location of emergency resources for traffic operation models and the basic model of sport mega-event are compared. The goal of the $P$-median model is to minimize the weighted distance, which is suitable for nonemergency emergency targets under normal circumstances, with relatively fixed emergency requirements and higher-cost emergency resource layouts, such as parks and gas stations. The optimization goal of the $P$-center model is to minimize the maximum distance. It is usually used in scenarios where the emergency goal is more urgent and emergency reliability is required, such as hospitals and fire stations; the main goal of the coverage model is to minimize the number of facilities to be constructed. Flexible location model is suitable for occasions with higher emergency requirements and is rarely used due to its poor cost control. Here, the emergency resource location models comparison table is shown in Table 1.

The flexible location of emergency resources model for traffic operation of sport mega-event has particularity and pertinence. First, the specificity of emergency requirements is guaranteed from the emergency time and materials. Second, the incremental layout of temporary emergency facilities + fixed emergency facilities meets the specificity of emergency facilities location and provides the possibility of compound rescue, reflecting its flexibility. This research model is suitable for the emergency resource layout of various large-scale mass events or major events with a higher security level. However, because it has no fixed cost constraints and poor economy, the emergency resource location in general situations is not applicable.

\section{Solution Algorithm of the Model}

To solve the emergency resource arrangement model of sports mega-event, the genetic algorithm with matrix realcoded is proposed. In the cross genetic segment, a modified optimal nearest neighbor crossover operator is designed to improve the convergence speed. The design idea is shown in Figure 1.
3.1. Limitation on the Quantity of Materials. Under the constraints of material reliability, the probability $\rho_{i}^{k}$ that emergency supplies can be provided is a monotonic increasing function of covering materials $s_{i}^{k}$ in demand points, that is, $f\left(s_{i}^{k}\right)$ [18]. The materials $s_{i}^{k}$ covering the demand points are integers, so $s_{i}^{k}$ satisfying the constraint conditions can be obtained by dichotomy.

Let $f^{\prime}\left(s_{i}^{k}\right)=f\left(s_{i}^{k}\right)-a$; therefore, $f^{\prime}(0)=-a<0$. On this basis, the specific steps of dichotomy are as follows:

(1) Firstly, let $n_{1}=0$ and $n_{2}=\sum_{i \in I} \operatorname{dem}_{i}^{k}$. Calculate $f^{\prime}\left(n_{1}\right)$ and $f^{\prime}\left(n_{2}\right)$, respectively.

(2) Let $n=\left|\left(n_{1}+n_{2}\right) / 2\right|$. Calculate $f^{\prime}(n)$.

(3) If $f^{\prime}\left(n_{1}\right) \cdot f(n+1) \leq 0$, then $s_{i}^{k}=n=1$, and end the algorithm; else, if $f^{\prime}\left(n_{1}\right) \cdot f(n+1)>0$, then $n_{1}=n$; if $f^{\prime}\left(n_{1}\right) \cdot f(n)<0$, then $n_{2}=n$.

(4) Repeat the cycle until the conditions are met.

(5) Algorithm termination.

3.2. Limitation on the Quantity of Minimum Time. Floyd algorithm is a classical planning algorithm, which uses dynamic programming to find the shortest path between the given weighted source points. The algorithm traverses any two points in the road network and sorts them step by step to get the shortest path matrix. The steps of the Floyd algorithm are as follows:

(1) Firstly, the weighted adjacency matrix $A$ is taken as the initial value of distance matrix $D$, that is, $D^{0}=A$.

(2) $D^{1}=d_{i j}^{(1)} n \times n, \quad d_{i j}^{(1)}=\min \left\{d_{i j}^{(0)}, d_{i 1}^{(0)}+d_{1 j}^{(0)}\right\}$ is the shortest length of the path from $v_{i}$ to $v_{j}$, of which passing point is only allowed to be $v_{1}$.

(3) In the same way, $D^{k}=d_{i j}^{(k)} n \times n$, $d_{i j}^{(k)}=\min \left\{d_{i j}^{(k-1)}, d_{i k}^{(k-1)}+d_{k j}^{(k-1)}\right\}$, is the shortest length of the path from $v_{i}$ to $v_{j}$, of which passing point is only allowed to be $v_{1}, v_{2} \ldots v_{k}$.

When $k=n D^{n}=d_{i j}^{(n)} n \times n, d_{i j}^{(k)}$ is the shortest path from $v_{1}$ to $v_{j}$ where any point can be inserted. Hence, $D^{(n)}$ is the distance matrix. In the iterative process, the matrix $R$ of the starting and succeeding points of the path can also be obtained, and the shortest path between the two points can be obtained from $R$. Finally, the shortest time matrix is obtained by dividing the average driving speed.

3.3. Modified Matrix Real-Coded Genetic Algorithm. The optimization of emergency resource arrangement is a typical NP-hard problem. Considering that GA has good performance in searching global optimal solution of multipeak and multidimensional problems, GA is selected to solve the model.

3.3.1. Coding and Initialization of Real Matrix. Real-coded can represent various combinations and expand the search scope of the understanding. Therefore, it has advantages in solving the variable problem of 


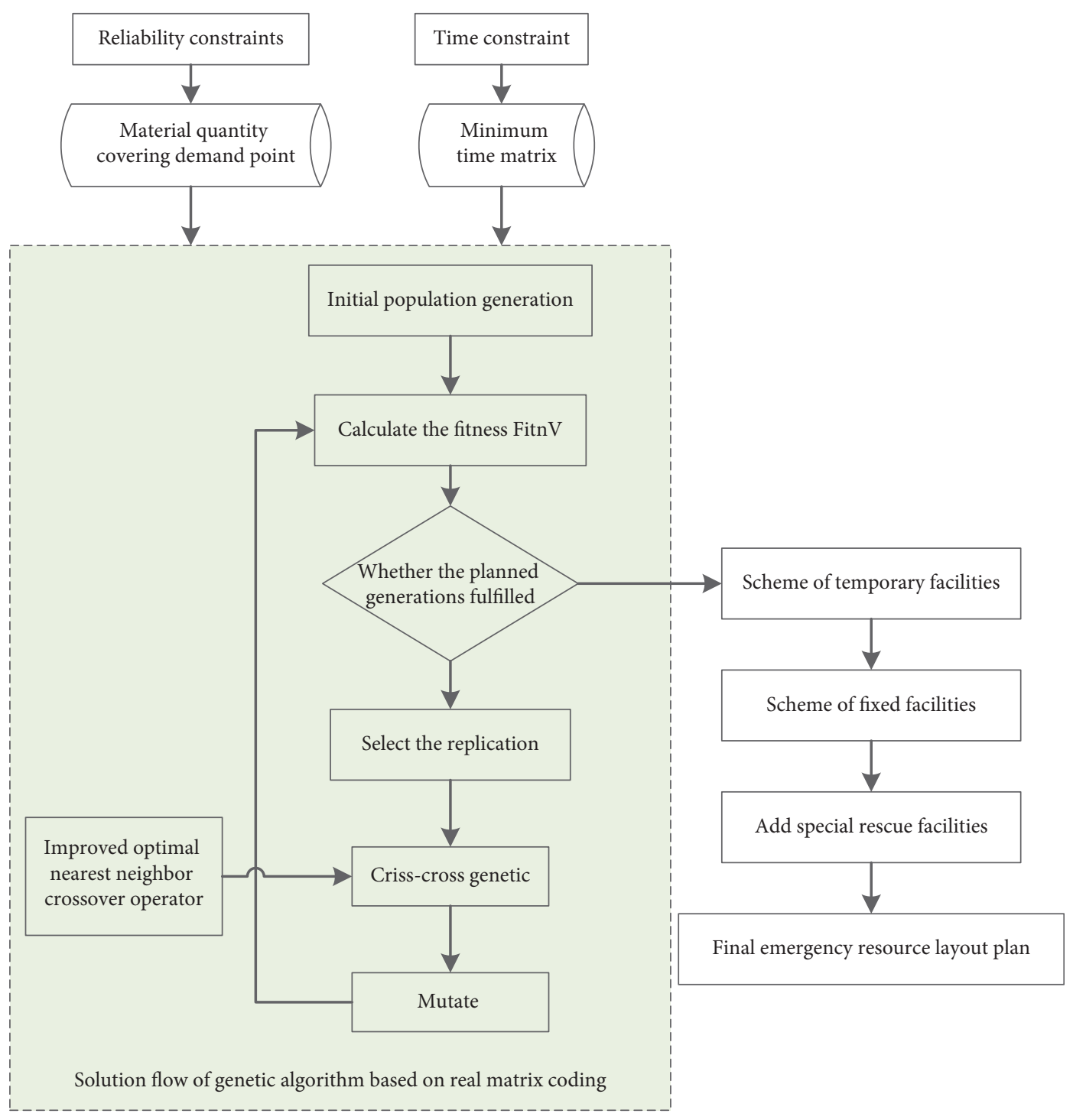

FIGURE 1: Flowchart of model solving algorithm.

multidimensional combination decision. The decision variables in this model are as follows:

$X_{j}$ (whether facilities are set up at $j$ ). When the total number of alternative facilities is $J$, the dimension of the variable of matrix $X_{1 \times J}$ is $1 \times J$.

$y_{j}^{k}$ (type- $k$ materials are placed in point $j$ facilities). When the types of materials are $K$, the dimension of the variable of matrix $X_{K \times J}$ is $K \times J$.

Because the two decision variables have the same number of columns, the two decision variables form a matrix to represent a single individual $R=\left(\begin{array}{c}X_{1 \times J} \\ X_{K \times J}\end{array}\right)$. Let the number of individuals in the initial population be $s$, each generation population can be expressed as $\mathrm{Ge}=\left\{R_{1}, R_{2} \ldots, R_{s}\right\}$, and the specific genotype of each individual is

$$
R_{s}=\left(\begin{array}{c}
X^{S} \\
Y^{S}
\end{array}\right)=\left[\begin{array}{cccc}
x_{1}^{s} & x_{2}^{s} & \ldots & x_{J}^{s} \\
y_{1}^{1 s} & y_{2}^{1 s} & \ldots & y_{J}^{1 s} \\
\vdots & \vdots & \ddots & \vdots \\
y_{1}^{K s} & y_{2}^{K s} & \ldots & y_{J}^{K s}
\end{array}\right]
$$

3.3.2. Improved Cross Genetic Segment. Because of the randomness of the sequential crossover operator, it is inevitable that some offspring have small fitness. When the road network is too large, the convergence speed will slow down and the operation time will be prolonged. According to the greedy algorithm, this paper designs an improved optimal nearest neighbor crossover operator; that is, in each operation loop, only the adjacent station with the highest fitness value is selected as the next arriving station. The 
improved crossover operator ensures that the hybrid offspring have higher fitness. The first step is to select the starting and ending positions of genes in a pair of chromosomes (parents); the second step is to randomly generate an offspring, calculate the position distance of the offspring and the adjacent genes, and compare with the parents; the third step is to select the genes with better distance and insert them into the corresponding positions. The flowchart is shown in Figure 2.

In the figure above, $a_{1}$ and $b_{1}$ are the subgenerations. $A_{1}=\left(a_{11}, a_{12}, \ldots, a_{1 n}\right)$ and $A_{2}=\left(a_{21}, a_{22}, \ldots, a_{2 n}\right)$ are parent generations of the generation. The distance between station points $a_{l 1}, a_{l 2}, a_{r 1}, a_{r 2}$, and $a$ on both sides is marked as $d_{1}, d_{2}, d_{3}, d_{4}$. Through the above flowchart, we can get the coding of the offspring $A_{1}$. Similarly, the offspring $A_{2}$ can be obtained by selecting a random crossover station $\beta$. The improved hybrid operator ensures that the hybrid offspring have a higher fitness value, so as to find the optimal solution with a faster convergence speed. Generally, the local optimal dilemma can be avoided by increasing mutation probability and using as many mutation types as possible.

\section{Case Study}

Based on the traffic organization during 2022 Beijing Winter Olympics, this paper selects Beijing Olympic Green as the sample area. Considering that the emergency resource arrangement for sports mega-events is wide in space and that the emergency resource arrangement for traffic operation should be flexible, the sample area is determined as follows: north to the 5th Ring Road of Beijing, south to the 3rd Ring Road of Beijing, west to Wanquan River Road, and east to Beijing Airport Expressway (as described in Figure 3).

4.1. Data Collection and Preprocessing. In view of the traffic contingency plan, we obtain the geographic information of medical, security, fire, and other facilities in the sample area. In this paper, we collect the relevant POI data through Amap's API open platform and Python.

The rescue time matrix $t_{i j}$ describes the rescue time that takes from any temporary emergency rescue facility location $j$ to any emergency demand point $i$ in the emergency area. Unlike the European distance used in previous studies, the rescue time matrix based on the actual road network distance is more in line with the reality of emergency rescue, and the layout is more reasonable. The key steps in calculating the matrix $t_{i j}$ are described as follows:

(1) A geodatabase is established which contains the set of emergency demand points, the set of temporary emergency facility alternatives, and the set of road networks.

(2) The shortest path matrix of each set is derived using the Floyd algorithm.

(3) According to the road conditions during megaevents and the designed speed for urban road (refer to Urban Road Engineering Design Specification (CJJ37-2012)), the bottom line of the designed speed for urban arterial roads, saying $40 \mathrm{~km} / \mathrm{h}$, is taken as the average speed. The rescue time matrix $t_{i j}$ is the shortest path matrix divided by the average speed.

\subsection{Parameters Setup. The parameters are assumed as} follows.

The cost of materials and labor, as well as the facility capacity, refers to the newsletter of 2010 Yushu earthquake (Qinghai, China) [19]. The maximum rescue time is drawn up with reference to the Traffic Service Documentary of Beijing Olympic Games and Paralympic Games, in which the half-hour service circle of emergency rescue is proposed. The construction cost of temporary facilities is set up to RMB 23,500 . In this paper, there are 296 emergency demand points and 100 temporary emergency facility alternatives.

The parameter assumptions depend on the emergency resource arrangement when the model is applied in practice. As our research is taken as an experiment, some parameters are simplified.

4.3. Results and Discussion. The matrix real-coded genetic algorithm is programmed using the Geatpy genetic algorithm toolbox for Python. This paper uses the roulette method with the parameters of the algorithm that set as follows: the default population is 100; the variation scale factor of differential evolution $F$ is 0.5 ; the cross probability $P_{C}$ is 0.5 ; the probability of variation $P_{m}$ is 0.1 ; and the maximum number of generations of the population is MAXGEN is 500 .

As shown in Figure 4, the target value of individuals varies with the number of generations. The solutions of the target function decrease with the number of generations and eventually stabilize. The optimal solution of the target function is $\min C=3032230$. That means the total system cost of emergency resource arrangement of sport events under the constraints of rescue time and resources. As can be seen from Figure 4, the matrix real-coded genetic algorithm can quickly converge to an optimal solution with the number of generations. Adding a nearest neighbor cross operator makes the model converge at a slightly faster rate than the conventional model.

The temporary emergency resource arrangement shows that 92 of the 100 alternatives are selected as the temporary emergency facility locations. $x_{4}, x_{9}, x_{28}, x_{36}, x_{42}, x_{85}, x_{87}, x_{98}$ are not selected for construction, so the allocation of supply is correspondingly 0 .

Based on the selection of temporary emergency facilities, the fixed emergency facilities are selected according to the ranking of the weighted distance. Along with the 92 selected temporary emergency facilities, a total of 26 existing facilities in the sample area are selected as fixed emergency facilities to meet the emergency demand in the sample area. The selected fixed emergency facility locations are shown in Table 5.

Using ArcGIS, the selected temporary and fixed emergency facilities as well as the stock of resources can be displayed on the visual geographic information map. As is shown in Figure 5, $H$ is marked as selected fixed emergency facilities, the yellow dots represent the selected temporary 


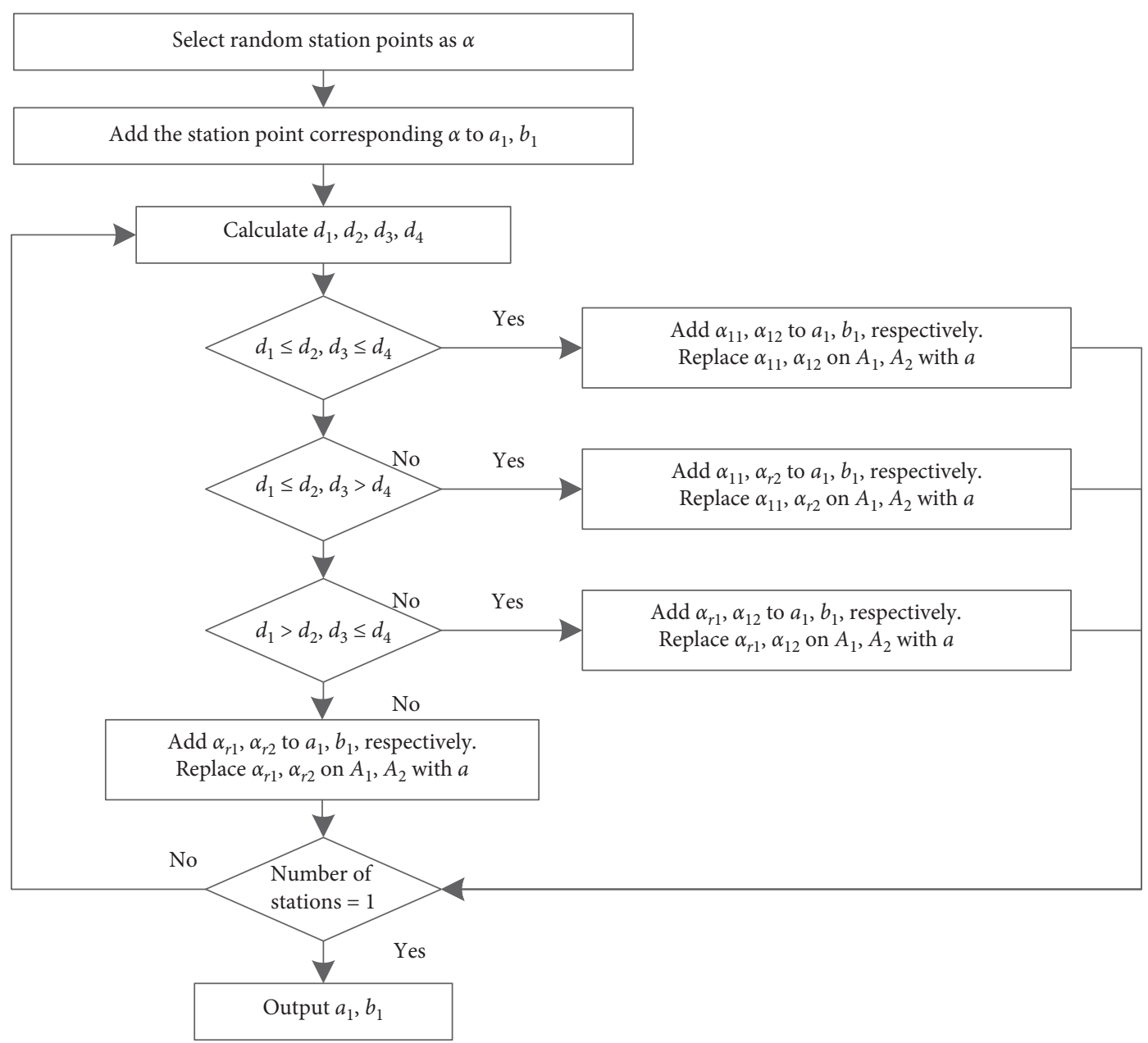

Figure 2: Flowchart of optimal nearest neighbor crossover operator.

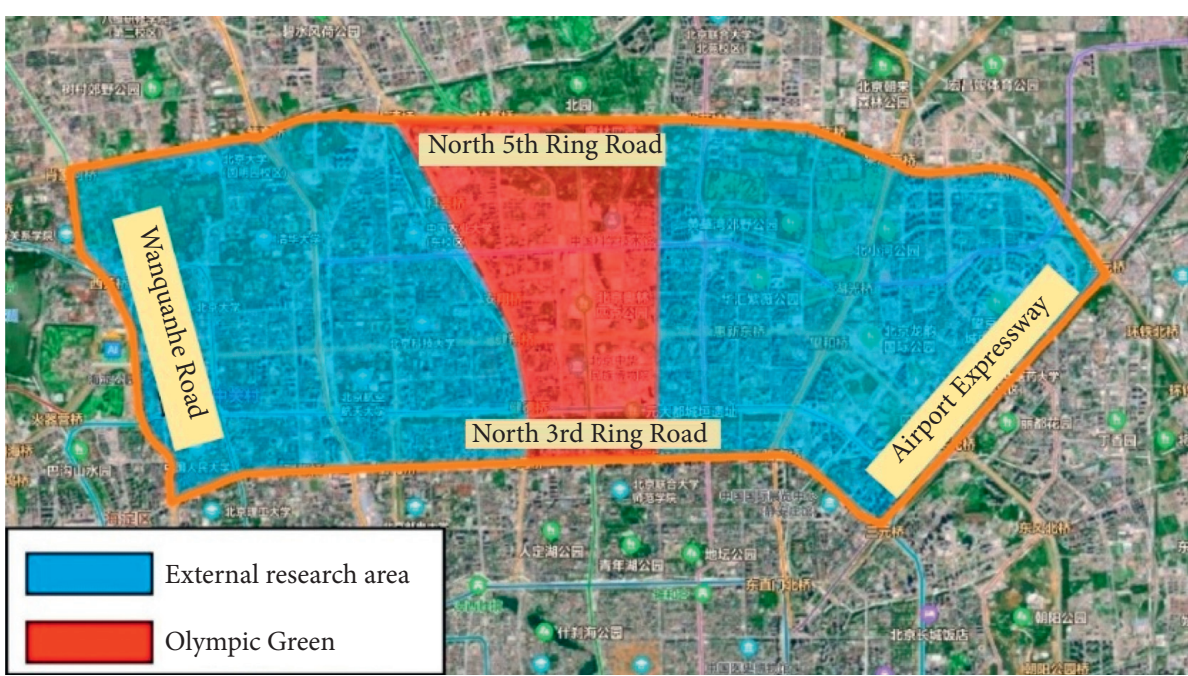

Figure 3: Research scope. 


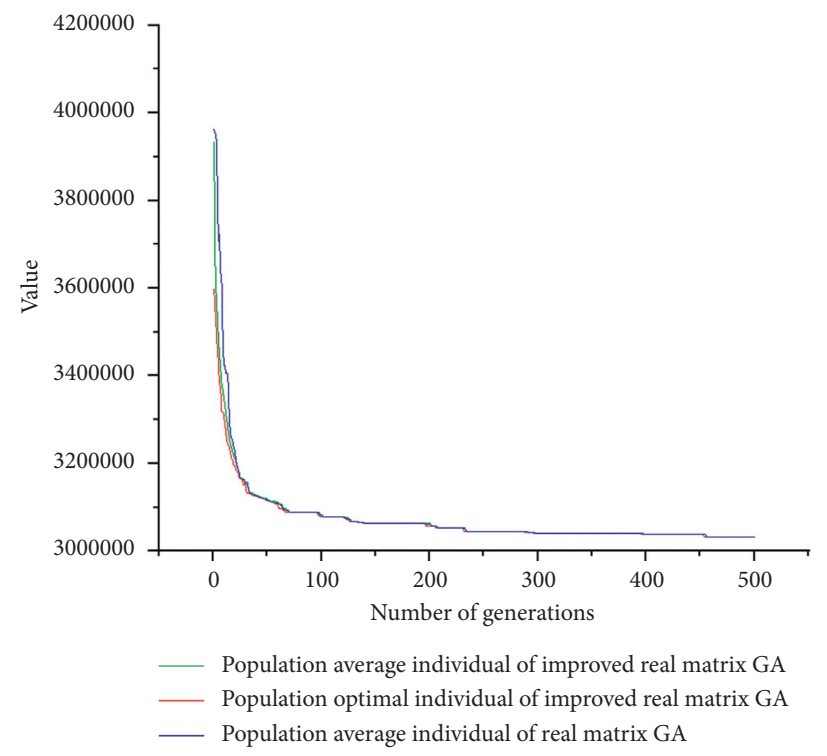

FIgURE 4: Value of individuals varies with the number of generations.

TABLE 5: Fixed emergency facility locations.

\begin{tabular}{|c|c|c|c|}
\hline ID & Name & Longitude & Latitude \\
\hline 0 & Peking University People's Hospital & 116.3479996 & 39.9351997 \\
\hline 1 & Peking University People's Hospital & 116.3479996 & 39.9351997 \\
\hline 2 & Beijing Shijitan Hospital & 116.3150024 & 39.8991013 \\
\hline 3 & East Medical Building & 116.2990036 & 39.924099 \\
\hline 4 & Beijing Hospital & 116.4089966 & 39.902401 \\
\hline 5 & Beijing Hospital & 116.4089966 & 39.902401 \\
\hline 6 & Beijing Organ Transplantation Center & 116.4469986 & 39.9248009 \\
\hline 7 & Beijing Center for Clinical Laboratory & 116.4469986 & 39.9248009 \\
\hline 8 & Beijing Rectum Hospital & 116.3740005 & 39.9581985 \\
\hline 9 & China-Japan Friendship Hospital (west courtyard area/warship hospital area) & 116.3619995 & 40.0085983 \\
\hline 10 & Xiyuan Hospital of CACMS & 116.288002 & 39.9939003 \\
\hline 11 & China PLA General Hospital of Rocket Army & 116.3659973 & 39.9552994 \\
\hline 12 & Wangjing Hospital of CACMS & 116.4670029 & 39.9812012 \\
\hline 13 & Beijing Ditan Hospital Capital Medical University & 116.5199966 & 40.0219002 \\
\hline 14 & Aviation General Hospital of China Medical University & 116.4140015 & 40.0278015 \\
\hline 15 & Wangjing Hospital of CACMS & 116.4670029 & 39.9812012 \\
\hline 16 & Beijing Ditan Hospital Capital Medical University & 116.5199966 & 40.0219002 \\
\hline 17 & Aviation General Hospital of China Medical University & 116.4140015 & 40.0278015 \\
\hline 18 & Beijing Hepingli Hospital & 116.4069977 & 39.9566994 \\
\hline 19 & Beijing Municipal Public Security Bureau (Dongcheng Branch) & 116.4039993 & 39.9365005 \\
\hline 20 & Beijing Municipal Public Security Bureau (Xicheng Branch) & 116.3600006 & 39.9095993 \\
\hline 21 & Beijing Municipal Public Security Bureau (Chaoyang Branch) & 116.4810028 & 39.9225998 \\
\hline 22 & Beijing Municipal Public Security Bureau (Haidian Branch) & 116.2939987 & 39.9589996 \\
\hline 23 & Shuangyushu Fire Squadron of Haidian District Public Security Fire Brigade & 116.3249969 & 39.9651985 \\
\hline 24 & Management Department, Beijing & 116.2839966 & 39.9455986 \\
\hline 25 & Eight Squadrons of Haidian Fire Brigade & 116.3349991 & 40.0051994 \\
\hline
\end{tabular}

emergency facilities, and the bar chart above each dot reflects the allocation of the two types of resources. Based on the results of data visualization, the temporary emergency facility locations have the following characteristics:

(1) The temporary emergency facility locations scatter and cover all the districts within the sample area to ensure the availability. As the central area of the layout, the Olympic Green is where frequent events happen, where the emergency demand is higher, and where the temporary emergency facility distribution is more intensive. On the other way, fewer temporary facilities are built outside the range of the Olympic Green.

(2) Temporary emergency facilities allocated with more resources are often located in the key routes with complex and intensive road networks, as the key 


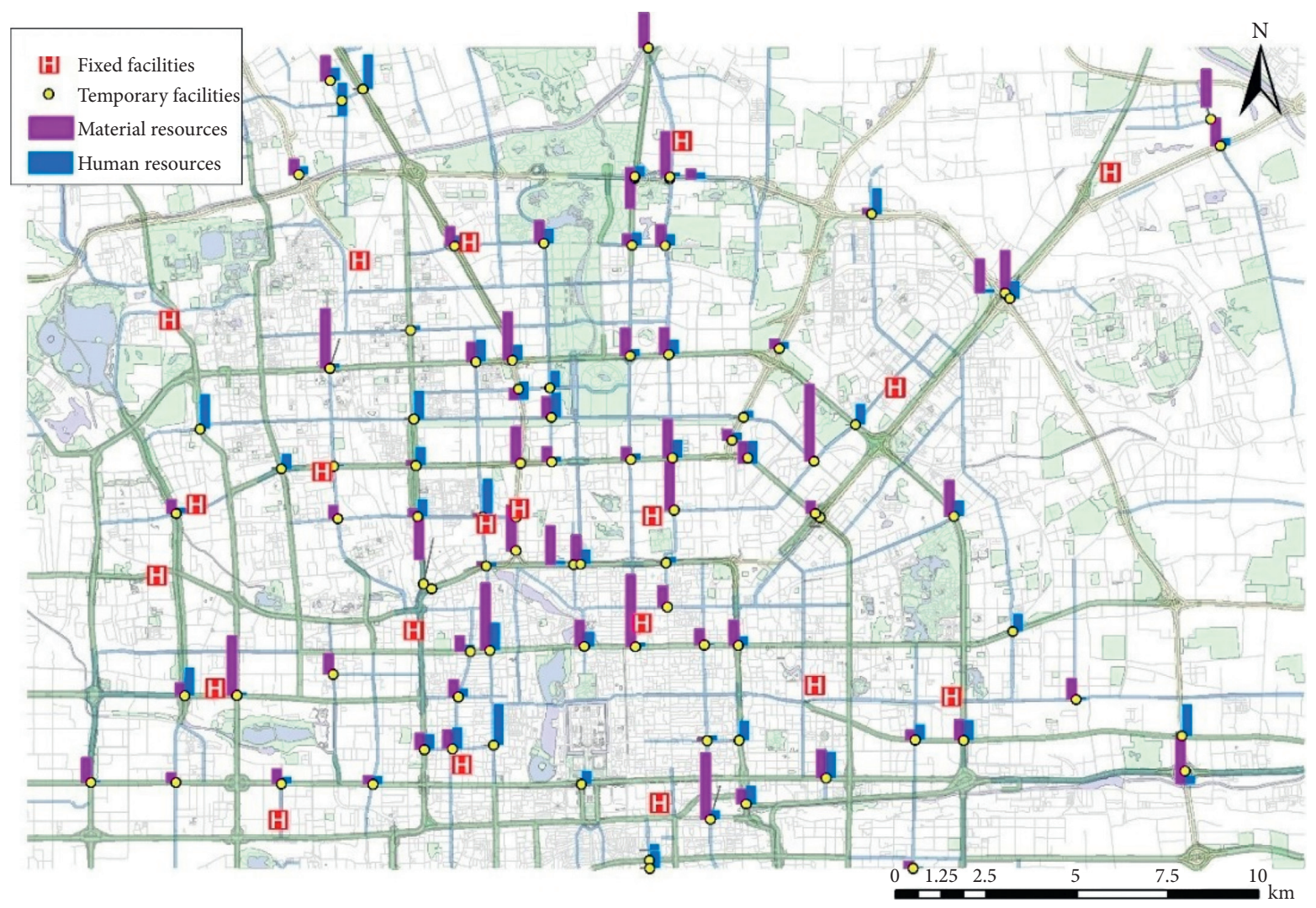

Figure 5: Visualization of results.

routes guarantee the operation of sport mega-events, where have a high demand for transportation and emergency. On the main road around these key routes, emergency resources are also adequate.

(3) In line with the objectives of the optimization model, the fixed emergency facility locations are distributed uniformly in the sample area and close to the temporary emergency facilities, because the fundamental objective of the fixed emergency facility locations is to provide the ongoing resources and special emergency needs (e.g., air rescue) to temporary facilities.

In a nutshell, the flexible arrangement that we obtain from the model generally meets the emergency demand in traffic operation of sport events. Besides, we consider the continuity of emergencies and the possibility of multiple rescue paths.

4.4. Further Analysis. A sensitivity analysis was performed in the deterministic case to understand the impact of the parameters that affect the objective value.

Among the preset parameters of Table 6, the probability of materials occupied $\mu^{1}$ and the probability of manpower occupied $\mu^{2}$ are unable to be constrained artificially. The preset value of $\mu^{1}$ and $\mu^{2}$ is more about the manager's confidence in the efficiency of material utilization. This paper studies the effect of $\mu^{1}$ change on the total cost. Set the value of $\mu^{1}$ from $30 \%$ to $70 \%$ in $10 \%$ steps, while keeping other parameters unchanged. The result is a monotonically increasing binary line graph. It can be seen that the total cost increases rapidly with the increase of $\mu^{1}$. This shows that when $\mu^{1}$ increases, managers need to alleviate this problem at a huge cost. The suggestions for the plan are to ensure sufficient materials and improve the efficiency of material operation. It can be predicted that when $\mu^{1}$ tends to $100 \%$, the cost of the whole model will also tend to be infinite.

The relationship between construction cost $C_{j}$, capacity $\mathrm{cap}_{j}$, and total cost is studied. There is no doubt that there is a positive correlation between $C_{j}$ and $\mathrm{cap}_{j}$. In order to facilitate the study, this paper assumes that it is a positive proportional relationship (i.e., land cost idealization) in a reasonable range. $C_{j}$ and $\operatorname{cap}_{j}$ are varied by $20 \%$ of their initial value synchronously, as shown in Figure 6 while keeping other parameters unchanged. And the relationships between them and total cost are projected on their respective coordinate systems. As can be seen from the figure, the broken line does not show obvious slope reduction. That is to say, there is no reduction in the required facilities, neither a slowdown in the growth trend of total cost, due to the larger capacity of facilities. This should be the result of the joint constraint of the shortest time matrix and the maximum rescue time threshold. The linear regression analysis of the projection on the coordinate system was carried out, and $R^{2}$ is 0.98 , which is a significant positive correlation. This shows that the constraint strength of the reliability of rescue time is significantly stronger than that of money cost in the model. This is in line with the background of large-scale sports events. 
TABle 6: Parameter assumptions.

\begin{tabular}{lc}
\hline Parameters & Value \\
\hline$C_{j}$ (construction cost of temporary facilities) & $\mathrm{RMB} 23,500$ \\
$v^{1}$ (volume of materials) & 0.5 units \\
$v^{2}$ (volume of labor) & 1 unit \\
$q^{1}$ (cost of materials) & $\mathrm{RMB} 100$ \\
$q^{2}$ (cost of labor) & $\mathrm{RMB} 150$ \\
cap (facility capacity) $_{T}$ (maximum rescue time) & 100 units \\
$\mu^{1}$ (probability of materials occupied) & $30 \mathrm{~min}$ \\
$\mu^{2}$ (probability of human occupied) & 0.4 \\
\hline
\end{tabular}

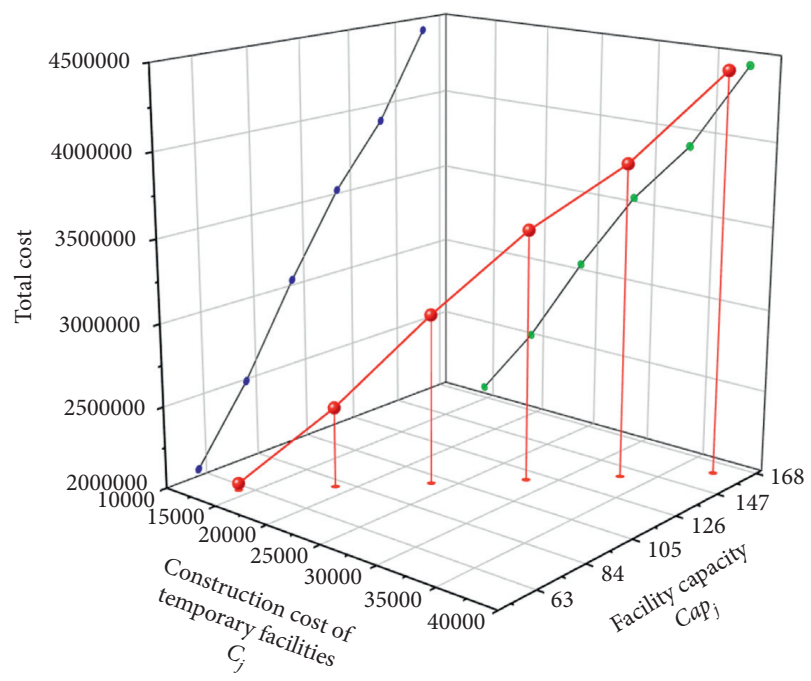

Figure 6: The graph of total cost, $C_{j}$, and $\operatorname{cap}_{j}$.

\section{Conclusion}

Emergency resource arrangement model is established for guaranteeing the traffic operation of sports mega-events and is solved by the modified matrix real-coded genetic algorithm.

This study gives a method to find weights of emergency recourses that equal weights of the road accident rates multiplying weights of the traffic service guarantee levels. Given the requirements of time reliability and resource adequacy in the traffic emergency, constraints of the time and resource reliability are added in the model. During sports events, temporary emergency facilities are set to ensure the operation and security of sport mega-events while fixed emergency facilities are set to take ground rescue and special air rescue and to transfer emergency resources among various types of facilities. Therefore, different from the arrangement of a single kind of facility in the previous, this paper simultaneously deploys fixed and temporary emergency facilities, which is more consistent with the realistic and thus has a more accurate result.

To obtain the travel time matrix, the shortest path algorithm is used here. Based on it and the reliability of resource allocation, the number of each kind of resource needed to be put in each facility location is calculated by the bisection method. On the basis, the modified matrix realcoded genetic algorithm is designed to solve the model. Because of the randomness of the sequential crossover operator, it is inevitable that some offsprings have small fitness, leading to a slow convergency. To deal with the problem, we design a nearest neighbor cross operator based on the greedy algorithm, which is later validated in the case to be efficient in helping the convergency of the algorithm.

The application of the model and algorithm is corroborated in the case of Beijing Olympic Green and its surrounding areas. We conduct the sensitivity analyzes of the probability of occupied materials, the construction cost, and the capacity. The results show that the probability of occupied materials has a great impact on the total cost. Events managers should ensure sufficient materials and improve the efficiency of material operation. Under the background of large-scale events, the impacts of construction cost and capacity on the total cost are significantly positively correlated; that is, the construction form and expenditure of emergency facilities will not affect the arrangement under the constraint of reliability.

\section{Data Availability}

The data of geographic information can be downloaded from the https://www.amap.com/. If the processed data are needed, please feel free to send an e-mail to author Shen Ling: shenlinglynn@qq.comshenlinglynn@qq.com.

\section{Conflicts of Interest}

The authors declare that they have no conflicts of interest.

\section{Acknowledgments}

This research work was supported by the Key R\&D Project of Jiangsu Province, Risk Identification, Prevention, and Control of Public Safety Events in Traffic Operation of Major Urban Events (BE2019713).

\section{References}

[1] M. Monika and G. Deepak, "Rapidly converging solution for p-centers in nonconvex regions," Turkish Journal of Electrical Engineering \& Uter Sciences, vol. 25, 2017.

[2] X. Menghao, Z. Jing, and Z. Qiuhong, "The research of location problem of national emergency reserve facilities for emergency relief based on P-center problem," Journal of Natural Disasters, vol. 28, no. 03, pp. 123-129, 2019.

[3] L. Özdamar and M. A. Ertem, "Models, solutions and enabling technologies in humanitarian logistics," European Journal of Operational Research, vol. 244, no. 1, pp. 55-65, 2015.

[4] F. Wang, Y. Hou, and S. Li, "Dynamic multiple coverage model of urban emergency material depository: from the perspective of supply and demand," Journal of China Agricultural University, vol. 023, no. 008, pp. 184-195, 2018.

[5] A. Kocatepe, E. E. Ozguven, M. Horner, and H. Ozel, "Pet-and special needs-friendly shelter planning in south Florida: a spatial capacitated P-median-based approach," International Journal of Disaster Risk Reduction, vol. 31, 2017. 
[6] C. Jian, H. Bo, and C. Shanzhi, "Resource allocation and location decision of a uav-relay for reliable emergency indoor communication," Computer Communications, vol. 159, 2020.

[7] L. Tie, B. Ruguo, and H. Jun, "A cost-efficiency equilibrium problem of regional single emergency resource guarantee with multi-objective programming," Systems Engineering Procedia, vol. 5, pp. 29-36, 2012.

[8] M. Yahyaei and A. Bozorgi-Amiri, "Robust reliable humanitarian relief network design: an integration of shelter and supply facility location," Annals of Operations Research, vol. 283, 2018.

[9] D. Jun, W. Jing, and Y. I. Xianqiang, "Study on lrp model and algorithm for emergency resource distribution after disaster," Journal of Safety Science and Technology, vol. 1, 2017.

[10] S. K. He and L. T. Liao, "An improved heuristics about layout of emergency station in a city," Advanced Materials Research, vol. 869-870, pp. 150-153, 2013.

[11] G. Barbarosogu and Y. Arda, "A two-stage stochastic programming framework for transportation planning in disaster response," Journal of the Operational Research Society, vol. 55, no. 1, pp. 43-53, 2004.

[12] R. Das and S. Hanaoka, "An agent-based model for resource allocation during relief distribution," Journal of Humanitarian Logistics and Supply Chain Management, vol. 4, no. 2, pp. 265-285, 2014.

[13] T. Zhaoping and S. Jianping, "Multi objective optimization of railway emergency rescue resource allocation and decision," International Journal of System Assurance Engineering and Management, vol. 9, 2018.

[14] H. O. Mete and Z. B. Zabinsky, "Stochastic optimization of medical supply location and distribution in disaster management," International Journal of Production Economics, vol. 126, no. 1, pp. 76-84, 2010.

[15] Z. Lingye, L. Jing, and Y. Zaili, "Optimal scheduling of emergency resources for major maritime oil spills considering time-varying demand and transportation networks," European Journal of Operational Research, vol. 293, no. 2, 2020.

[16] R. Church and C. R. Velle, "The maximal covering location problem," Papers in Regional Science, vol. 32, no. 1, pp. 101-118, 2005.

[17] Q. Jin, Y. E. Yong, S. Chunyan, Z. Wei, and H. Jian, "Optimization method for emergency resource layout for transportation network considering service reliability," Journal of Railway Ence and Engineering, vol. 15, no. 2, pp. 506-514, 2018.

[18] C. Fang-Fang, D. Xiao-Mei, and W. Sheng-Yong, "Emergency management of yushu earthquake tests the wenchuan experience," The Journal of Evidence-Based Medicine, vol. 10, no. 2, pp. 157-162, 2010.

[19] D. Konur, M. M. Golias, and B. Darks, "A mathematical modeling approach to resource allocation for railroad-highway crossing safety upgrades," Accident Analysis \& Prevention, vol. 51, pp. 192-201, 2013. 